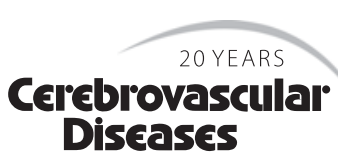

\title{
The Johann Jakob Wepfer Award 2010 of the European Stroke Conference to Professor Jan van Gijn
}

\section{Background and Introduction}

How should one summarise the career of such a major scholar, scientist and clinician as Jan van Gijn? Not an easy task, because he is in many ways a 'Renaissance man' with very wide-ranging scholarly interests. His interests include (but are not limited to): the history of medicine; the fundamentals of bedside neurological practice; the treatment and prevention of stroke and subarachnoid haemorrhage; a variety of other neurological disorders, and, throughout his work, a keen interest in the science of evidence-based medicine (randomised trials, systematic reviews and high-quality observational epidemiological studies).

\section{Methods}

The ideal approach to providing a brief, but unbiased summary of such a large canon of work is to adopt the methods of the Cochrane Collaboration and undertake a systematic, evidencebased review. Such reviews, of course, begin with a formal statement of the methods to be used. To prepare this review, I adopted a formal search strategy on the variations of Jan's name that one might find in bibliographic databases (the terms used were: van Gijn J OR Vangijn J OR Gijn JV). The strategy was applied to Medline, the Web of Science and supplemented with searches of Google Scholar, Google Images and Wikipedia. The results of the search were then submitted to both quantitative and qualitative analyses with application of statistical tests of significance and confidence intervals, where appropriate. The intention was to conclude with a summary of implications for practice and impli- cations for research. Cochrane reviews are, of course, subject to a process of rigorous external peer review. After a relatively brief search I was able to identify a wise old neurologist in the misty hills of Scotland (himself a previous Johann Jakob Wepfer Award winner), Charles Picton Warlow, who provided valuable external peer review.

\section{Quantitative Results}

The quantitative analysis of the Web of Science yielded 422 scientific publications attributed to Jan between 1969 and 2010. These articles have been cited in excess of 18,900 times. Many of the articles have been in journals with extremely high impact factors. The Web of Science classified the papers as covering an extraordinarily wide range of domains (the number in brackets that follows each subject is the number of publications covering that field): clinical neurology (328), peripheral vascular disease (127), surgery (93), medicine, general and internal (64), psychiatry (61), neurosciences (30), radiology and medical imaging (10), neuroimaging (6), haematology (5), cardiac and cardiovascular systems (4) ... and the list goes on. His most recent publication in 2010 relates to very interesting observations on the nature of chronic pain, a subject which is becoming increasingly important in the field of stroke rehabilitation. The quantitative analysis depends very much on the question being asked. If one asks the artificial question 'is van Gijn a better scientist than Wepfer?', it would seem reasonable to apply a test of statistical significance to the number of citations of the

\section{KARGER}

Fax +4161306 1234 E-Mail karger@karger.ch www.karger.com
(C) 2010 S. Karger AG, Basel

1015-9770/10/0304-0327\$26.00/0 
author's publications on the Web of Science. The number of citations for Jan van Gijn is 18,900, and the number for Wepfer (his historical control) is 0 . This is a highly statistically significant result $(\mathrm{p}<0.00001)$, a result sufficiently robust that confidence intervals are not informative. What should one conclude from this? Firstly, that studies involving historical controls are invariably unreliable. Secondly, citation metrics are not the whole story in assessing the merit of individual scientists (yet sadly, many institutions today are obsessed with such numbers). However, it is clear - on pure common sense grounds alone - that both van Gijn and Wepfer should be judged as outstanding scientists of their time.

And what of the qualitative analysis of the life and career of van Gijn? A search of Google Images for 'van Gijn' produced a rather delightful image from the website of Erasmus University, Rotterdam, in which the publication topics of the papers that van Gijn had conducted in collaboration with colleagues in Rotterdam were displayed as 'word art' that conveyed in a rather different way the breadth of topics in which he has demonstrated scholarly excellence (I suggest you go to Google Images and repeat the search for yourself).

A clinical topic of Jan's research - which might at first sight not seem to be a subject of huge scientific importance - is the Babinski sign. This was the subject of Jan's MD thesis, and a series of publications and a monograph (published in 1996) that followed. Jan approached this subject from an interesting dual perspective. Firstly, through the eyes of a medical historian and secondly, as a clinician applying the methods of evidence-based medicine of the 20th century to evaluate the validity and reliability of the sign. Babinski had been interested in identifying physical signs that could separate functional hemiparesis from weakness due to organic disease of the nervous system. It is quite humbling to realise that, even now in 2010, in the era of highly advanced brain imaging, careful clinical assessment of the Babinski sign (applying the test as described by van Gijn) can still provide useful confirmatory evidence about whether a patient's weakness is organic or functional. This has particular resonance in the era of thrombolytic treatment when one surely wishes to avoid unnecessary treatment with recombinant tissue plasminogen activator (which can occasionally trigger really serious allergic reactions) in a patient presenting with an acute hemiparesis which is functional and not due to ischaemic cerebrovascular disease.

Truly original observations in clinical medicine are rare, and ones that make a really substantial impact on patient management even rarer. Jan noted that there was a group of patients with subarachnoid haemorrhage and negative angiograms that tended to have a pattern of blood distribution around the midbrain. Patients with this clinical syndrome, subsequently named perimesencephalic haemorrhage (in an earlier era it might have been named the Van Gijn Syndrome), have an extremely benign prognosis, almost always require only a single angiogram and - even though the cause is not precisely known - can be reassured, discharged home and managed accordingly. Appropriate recognition of this subset of patients with subarachnoid haemorrhage is now an important part of clinical practice in the field.

Jan van Gijn is, above all, a very humane clinician and there are many examples in his published work of his compassionate, humane, humble (and sometimes selfdeprecating) approach to clinical medicine. One illustrative example is a case report he wrote for Charles Warlow's journal Practical Neurology in 2006. He described talking to a patient who was reporting the problems she had from her sensory peripheral neuropathy. He was just about to say - in front of the assembled ward round group - 'I know how you feel' when he paused for reflection. He remembered what had happened that very morning. As he entered the ward, a nurse had pointed out that he was still wearing his cycle clips! He himself has a mild sensory neuropathy and, as a result, was completely unaware of the clips on his legs. He then used this brief interaction with the patient to illustrate - from a patient's perspective - some of the unusual problems and symptoms that a patient with neuropathy may experience that neurologists should be aware of in their daily practice.

Jan is also a passionate and highly skilled teacher. $\mathrm{He}$ wrote in the Dutch Medical Journal in 2010, deprecating the radical change in teaching methods that had come in the 21st century. 'The style of teaching in small groups led by junior staff who have no specialised knowledge but act only as a supervisor while students teach each other' was, in his view, 'a shoddy substitute because teaching is an art and because students cherish proper explanations'. There are some other themes which run through Jan's life and work: a strong commitment to family, a commitment to collaboration (notably his support for collaborative research projects), his enduring commitment to clinical trials, especially investigator-led clinical trials, and his high scientific integrity. His lectures and presentations are always a model of clarity, and a feature of his lectures is the inclusion of relevant artworks (to lighten some of the drier aspects of evidence-based medicine) and - often - a picture of his home town Utrecht (the city's Dom tower appears frequently). 


\section{Conclusions: Implications for Clinical Practice}

In his time, Jan's scientific publications have given us the evidence on, for example:

(1) lower-dose aspirin for stroke prevention (avoiding many of the toxic side effects associated with higher doses),

(2) the need to avoid oral anticoagulants in patients with ischaemic stroke of arterial origin, and

(3) the importance of recognising perimesencephalic haemorrhages and treating them appropriately.

\section{Conclusions: Implications for Research}

His work has yielded the following implications for research:

(1) it has underpinned efforts to improve evidence-based stroke care and the quality of stroke research,

(2) he has trained a cohort of clinician scientists to undertake the investigator-led trials of the future, and

(3) perhaps most importantly, one hopes that all of us will, in future, aspire to maintain the high standards of design, conduct, analysis, reporting and publishing of scientific clinical research that he has set during his career.

Peter Sandercock, Edinburgh 